PROCEEDINGS OF THE

AMERICAN MATHEMATICAL SOCIETY

Volume 133, Number 8, Pages 2251-2258

S 0002-9939(05)07815-9

Article electronically published on March 15, 2005

\title{
E-ALGEBRAS WHOSE TORSION PART IS NOT CYCLIC
}

\author{
GÁBOR BRAUN AND RÜDIGER GÖBEL
}

(Communicated by Bernd Ulrich)

\begin{abstract}
We consider algebras $A$ over a Dedekind domain $R$ with the property $A \cong \operatorname{EndAlg}_{R} A$ and generalize Schultz' structure theory of the case $R=\mathbb{Z}$ to Dedekind domains. We construct examples of mixed $E(R)$-algebras, which are non-split extensions of the submodule of elements infinitely divisible by the relevant prime ideals. This is also new in the case $R=\mathbb{Z}$.
\end{abstract}

\section{INTRODUCTION}

1.1. Notion of an $E(R)$-algebra. Let $R$ be a commutative ring and $M$ an $R$ module. The endomorphism ring End $M$ of $M$ is an $R$-algebra acting on $M$ on the right. We will denote the endomorphism ring by Endo $M$ if we think of it as an $R$-module.

If $M$ and Endo $M$ are isomorphic, then $M$ is called an $E(R)$-module.

If $A$ is an $R$-algebra, then $\mu: A \rightarrow$ End $A$ mapping $a \in A$ to right multiplication by $a$ is an algebra homomorphism. If $\mu$ is an isomorphism, then $A$ is an $E(R)$ algebra.

An $R$-algebra $A$ is a generalized $E(R)$-algebra if it is isomorphic to End $A$.

Note that $E(R)$-algebras are precisely the commutative generalized $E(R)$-algebras. Every generalized $E(R)$-algebra is an $E(R)$-module, and every $E(R)$-module admits a generalized $E(R)$-algebra structure, which is unique up to isomorphism. Thus $E(R)$-modules and generalized $E(R)$-algebras are essentially the same.

The above notions are generalizations of $E$-rings, i.e. $E(\mathbb{Z})$-algebras.

These notions go back to Schultz; see [5]. Examples of $E(R)$-modules are given in Section 2 .

1.2. Main results. This subsection summarizes our main results, which will be proved in later sections.

From now on let $R$ be a Dedekind domain. Let $Q$ denote the quotient field of $R$. If $S$ is a set of prime ideals of $R$, then an $R$-module $M$ is $S$-divisible if $M=P M$ for all $P \in S$. The module $M$ is $S$-reduced if it has no non-trivial $S$ divisible submodule. If $N \subseteq M$, then $N$ is $S$-pure in $M$ (denoted by $N \subseteq S M$ ) if $I N=N \cap I M$ for all ideals $I$ of $R$ which are products of prime ideals from $S$.

Received by the editors February 17, 2003 and, in revised form, July 22, 2003 and April 20, 2004.

2000 Mathematics Subject Classification. Primary 16W20; Secondary 16D70.

Key words and phrases. Mixed E-rings, Dedekind domain.

This work was supported by the project No. I-706-54.6/2001 of the German-Israeli Foundation for Scientific Research \& Development.

(C)2005 American Mathematical Society Reverts to public domain 28 years from publication 
For example, $T:=\bigoplus_{P \in S} R / P^{n_{P}}$ is an $S$-pure submodule of

$$
\Pi:=\prod_{P \in S} R / P^{n_{P}}
$$

where the $n_{P}$ are arbitrary positive integers. Both $T$ and $\Pi$ are $S$-reduced, and they are divisible by all prime ideals not in $S$. Note that $\Pi / T$ is divisible: if $Q \in S$, then $\Pi / T$ is a factor of the $Q$-divisible module $\Pi:=\prod_{P \in S \backslash\{Q\}} R / P^{n_{P}}$ and hence it is $Q$-divisible. If $Q \notin S$, then $\Pi / T$ is $Q$-divisible since $\Pi$ is already $Q$-divisible.

Now we can reformulate the structure theory of $E$-rings from Schultz 5, p. 63, Theorem 6] for Dedekind domains.

Theorem 1.1. Let $A$ be a generalized $E(R)$-algebra. Then

(i) The primary components of the torsion part $T$ of $A$ are cyclic:

$$
T=\bigoplus_{P \in S} R / P^{n_{P}}
$$

where $S$ is a set of prime ideals of $R$ and the $n_{P}$ are positive integers. After Schultz, the elements of $S$ are called the relevant prime ideals.

(ii) Using the above notation, let $D$ be the largest $S$-divisible submodule of $A$. Then $D$ is an ideal, $A / D$ is an $S$-reduced subalgebra of $\prod_{P \in S} R / P^{n_{P}}$ and

$$
T=\bigoplus_{P \in S} R / P^{n_{P}} \subseteq_{S} A / D \subseteq S \prod_{P \in S} R / P^{n_{P}}=: \Pi .
$$

Using the notation and assumptions of the previous theorem, we have a pull-back diagram:

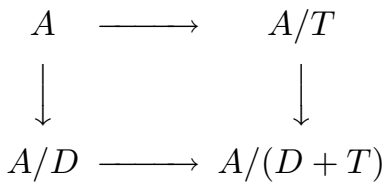

This suggests that we can construct mixed $E(R)$-algebras as pull-backs; see Lemma 4.1. It is a fruitful method, as it gives the first example of an $E(R)$-module $A$ for which $D$ is not a direct summand as stated in the next theorem. Let $R_{S}$ be the localization of $R$ at the set $S$ of prime ideals.

Theorem 1.2. Let $S$ be an infinite set of prime ideals of $R$. Suppose that $S$ does not contain all the prime ideals and $R_{S}$ is not a complete discrete valuation ring. Then for any family $\left\{n_{P}: P \in S\right\}$ of positive integers there exists an arbitrarily large $E(R)$-algebra $A$ whose torsion submodule is given by (1) and whose $S$-divisible part $D$ is not a direct summand.

This indicates that the classification of mixed $E(R)$-modules is a difficult task. However, the classification of non-reduced $E$-rings generalizes to $E(R)$-algebras:

Theorem 1.3. The non-reduced $E(R)$-modules over a Dedekind domain $R$ are exactly the $R$-modules of the form $Q \oplus C$ where $C$ is a torsion cyclic $R$-module. In particular they are all $E(R)$-algebras. 


\section{Examples of $E(R)$-MOdules}

2.1. Torsion-free $E(R)$-modules. Though most of the references below deal only with the case $R=\mathbb{Z}$, their methods generalize without problem to Dedekind domains $R$ which are neither fields nor complete discrete valuation domains. Therefore we state the results for Dedekind domains.

If $R$ is a field, then $R$ is also the only $E(R)$-algebra. Let us note that all $E(R)$ algebras over a complete discrete valuation domain are $R, Q(R)$ and the direct sums $Q(R) \oplus R / p^{n} R$ for all $n \in \mathbb{N}$, so the exclusion of these rings is necessary. We also note that the generalized $E(R)$-algebras over a complete discrete valuation domain are $E(R)$-algebras; see [3]. Thus it is also necessary to exclude these rings.

Classical examples of torsion-free $E(R)$-algebras are the subalgebras of $Q$ and the pure subalgebras of $J_{P}$, the algebra of $P$-adic integers over $R$ where $P$ is a prime ideal.

Arbitrarily large $E(R)$-algebras were constructed using the Black Box in Dugas, Mader, Vinsonhaler [1]. The proof can be simplified by use of the Strong Black Box; see [4] and also [3]. These $E(R)$-algebras are distorted polynomial algebras over $R$.

Finally, we mention some more constructions of E-rings. Faticoni 2] showed that every countable reduced torsion-free commutative ring is a pure subring of an $E$-ring. The same is true for rings of cardinality $\leq 2^{\aleph_{0}}$; see [3].

2.2. $E(R)$-modules whose torsion part is a direct summand. An $R$-module is an $E(R)$-module whose torsion part is a direct summand iff, for some non-zero ideal $I$ of $R$, it is of the form $R / I \oplus N$ where $N$ is a torsion-free $I$-divisible $E(R)$ module. ( $N$ is $I$-divisible means $N=I N$.) This follows directly from Theorem 1.1

Thus this class of $E(R)$-modules is classified modulo torsion-free $E(R)$-modules. In particular, the torsion $E(R)$-modules are precisely the cyclic modules.

2.3. Other mixed $E(R)$-modules. The following example is due to Schultz. Let $S$ be a set of prime ideals of $R$. Let $A$ be a subalgebra of $\prod_{P \in S} R / P^{n_{P}}$ such that

$$
\bigoplus_{P \in S} R / P^{n_{P}} \subseteq_{S} A \subseteq S \prod_{P \in S} R / P^{n_{P}} .
$$

Then $A$ is an $E(R)$-algebra and $S$ is the set of relevant prime ideals.

So far we have only seen $E(R)$-modules whose $S$-divisible part is a direct summand. But this is not true for our examples in Section 4

Mixed $E(R)$-modules can be constructed as a pull-back of the last example and a torsion-free $E(R)$-module. See Lemma 4.1 for the precise construction.

\section{The Structure theory of $E(R)$-ALGEBras}

In this section we prove Theorems 1.1 and 1.3, which describe the structure of $E(R)$-algebras.

We will use $N \sqsubseteq M$ to denote that $N$ is a direct summand of $M$ as an $R$-module.

We recall some well-known facts about torsion submodules over Dedekind domains used below. Torsion modules decompose into primary components. Each torsion module $M$ has a basic submodule i.e. a pure submodule $B$ which is a direct sum of cyclic modules and $M / B$ is divisible. 
Basic submodules of $M$ are not unique but the number of cyclic summands $R / P^{k}$ is unique for all $k$. For example, this number is the largest cardinal $\kappa$ for which $\left(R / P^{k}\right)^{(\kappa)}$ is a direct summand of $M$.

Divisible submodules and pure bounded submodules are direct summands in any module. Divisible modules are direct sums of modules $R_{P \infty}$ 's and $Q$. The number of the summands $R_{P \infty}$, respectively $Q$ is independent of the decomposition. Namely, in every decomposition of a divisible module $M$, the number of the summands $Q$ is the largest cardinal $\kappa$ for which $Q^{(\kappa)}$ is a direct summand of $M$. The same result holds for $R_{P \infty}$.

Recall that $J_{P} \cong$ End $R_{P^{\infty}}$ is the algebra of $P$-adic integers over $R$. This gives an embedding of $R_{P \infty}$ in $\operatorname{Hom}\left(J_{P}, R_{P \infty}\right)$. As we already mentioned, $J_{P} \cong$ End $J_{P}$.

Recall that $\operatorname{Hom}(A, B)$ is torsion-free if $B$ is divisible, and $\operatorname{Hom}(A, B)$ is divisible if $A$ is torsion-free and $B$ is divisible.

The proofs of the next two lemmas are obvious.

Lemma 3.1. Let $M$ be a torsion cyclic module or the quotient field $Q$. Then for every cardinal $\kappa$,

$$
\text { Endo } M^{(\kappa)} \cong M^{(\lambda)}
$$

for some $\lambda$. If $\kappa>1$, then $\lambda>\kappa$.

Lemma 3.2. Let $A$ be an $E(R)$-module. Suppose that $A=B \oplus C$ is a direct sum of $R$-modules. Then Endo $B \oplus$ Endo $C$ is a direct summand of $A$ as an $R$-module. Moreover,

$$
A \cong \text { Endo } A=\text { Endo } B \oplus \operatorname{Hom}(B, C) \oplus \operatorname{Hom}(C, B) \oplus \text { Endo } C .
$$

3.1. The main structure theorem. We are ready to prove Theorem 1.1. The two parts of the theorem are proven separately.

Proof of Theorem 1.1(i). By the preceding remarks, a basic submodule of the $P$ component $A_{P}$ of $A$ is a direct sum of cyclic $P$-modules: $\bigoplus_{k=1}^{\infty}\left(R / P^{k}\right)^{(\kappa(k))}$ where $\kappa(k)$ is the largest cardinal $\kappa$ for which $R_{P^{k}}^{(\kappa)}$ is a direct summand of $A$. First we prove that $\kappa(k)$ is at most 1 .

From Lemmas 3.1 and 3.2 we have a direct summand $R_{P^{k}}^{(\lambda)}$ of $A$ where $\lambda>\kappa(k)$ if $\kappa(k)>1$. Hence $\kappa(k) \leq 1$.

Now we prove that the basic subgroup of $A_{P}$ is cyclic. Otherwise $R_{P^{k}} \oplus R_{P^{l}}$ would be a direct summand of $A$ for some $k<l$. Then

(7) $\operatorname{Endo}\left(R_{P^{k}} \oplus R_{P^{l}}\right)=\operatorname{Endo} R_{P^{k}} \oplus \operatorname{Hom}\left(R_{P^{k}}, R_{P^{l}}\right) \oplus \operatorname{Hom}\left(R_{P^{l}}, R_{P^{k}}\right) \oplus \operatorname{Endo} R_{P^{l}}$

is a direct summand of $A$ isomorphic to $R_{P^{k}}^{3} \oplus R_{P^{l}}$, contradicting the previous paragraph. Hence the basic submodule of the $P$-component is cyclic.

It follows that $A_{P}$ is a direct sum of a cyclic $P$-module (a basic submodule) and a divisible one $R_{P^{\infty}}^{(\kappa)}$, where $\kappa$ is maximal with $R_{P^{\infty}}^{(\kappa)}$ being a direct summand of $A$. We show that $\kappa=0$ and hence $A_{P}$ is cyclic.

By a double application of Lemma 3.2

$$
\operatorname{Hom}\left(\text { Endo } R_{P^{\infty}}^{(\kappa)}, R_{P^{\infty}}^{(\kappa)}\right) \sqsubseteq A .
$$

Now Endo $R_{P^{\infty}}^{(\kappa)}$ is torsion-free since $R_{P^{\infty}}^{(\kappa)}$ is divisible. Hence

$$
\operatorname{Hom}\left(\text { Endo } R_{P^{\infty}}^{(\kappa)}, R_{P^{\infty}}^{(\kappa)}\right)
$$


is divisible. Its $P$-divisible part is $R_{P^{\infty}}^{(\lambda)}$, where $\lambda$ is the dimension of the $P$-socle:

$$
\operatorname{Hom}\left(\text { Endo } R_{P \infty}^{(\kappa)}, R_{P \infty}^{(\kappa)}\right)[P] \cong \operatorname{Hom}\left(\left(\text { Endo } R_{P \infty}^{(\kappa)}\right) \otimes R / P, R_{P}^{(\kappa)}\right) \sqsupseteq \operatorname{Endo} R_{P}^{(\kappa)} \text {. }
$$

The dimension of (Endo $\left.R_{P \infty}^{(\kappa)}\right) \otimes R / P$ over $R / P$ is obviously at least $\kappa$ (e.g. since the canonical projections to the coordinates are linearly independent), which justifies the last relation in (9). If $\kappa>1$, then we have $\lambda>\kappa$ since the dimension of Endo $R_{P}^{(\kappa)}$ is larger than $\kappa$ by Lemma 3.1. But $\lambda>\kappa$ contradicts the maximality of $\kappa$. Thus $\kappa \leq 1$.

We also have to exclude the case $\kappa=1$. So suppose that $R_{P \infty} \sqsubseteq A$. Then $J_{P} \cong$ Endo $R_{P \infty} \sqsubseteq A$. So $R_{P^{\infty}} \oplus J_{P} \sqsubseteq A$. By Lemma 3.2

$$
J_{P} \oplus J_{P} \cong \text { Endo } R_{P \infty} \oplus \text { Endo } J_{P} \sqsubseteq A \text {. }
$$

Hence $R_{P \infty}^{2} \sqsubseteq \operatorname{Hom}\left(J_{P}^{2}, R_{P \infty}\right) \sqsubseteq A$, which is impossible.

So every $P$-component is reduced with cyclic basic submodule; hence all of them are cyclic.

Now we are ready to prove the second part of the main structure theorem.

Proof of Theorem 1.1(iil). If $P \in S$, then the $P$-component $R / P^{n_{P}}$ of $A$ is cyclic and pure in $A$. Hence it is a direct summand:

$$
A=R / P^{n_{P}} \oplus B_{P} .
$$

Thus we have

$A \cong$ Endo $A=$ Endo $R / P^{n_{P}} \oplus \operatorname{Hom}\left(R / P^{n_{P}}, B_{P}\right) \oplus \operatorname{Hom}\left(B_{P}, R / P^{n_{P}}\right) \oplus$ Endo $B_{P}$.

The first three summands are all $P$-modules. But Endo $R / P^{n_{P}} \cong R / P^{n_{P}}$, so this is the whole $P$-component of $A$.

In particular, we must have $\operatorname{Hom}\left(B_{P}, R / P^{n_{P}}\right)=0$ so $B_{P}$ is $P$-divisible.

It follows that $B_{P}$ is the largest $P$-divisible submodule of $A$ and hence is an ideal of $A$. Also, $R / P^{n_{P}}$ is an ideal of $A$, being the $P$-torsion part of $A$. So $A=R / P^{n_{P}} \times B_{P}$ as $R$-algebras and this gives rise to an algebra epimorphism $\varphi_{P}: A \rightarrow R / P^{n_{P}}$.

Combining the algebra homomorphisms $\varphi_{P}$ together for all $P \in S$, we obtain a homomorphism

$$
\varphi: A \rightarrow \prod_{P \in S} R / P^{n_{P}}
$$

Obviously, $\varphi$ restricted to the torsion part $T$ of $A$ is just the canonical embedding of $T=\bigoplus_{P \in S} R / P^{n_{P}}$ into $\prod_{P \in S} R / P^{n_{P}}$.

Let $D:=\bigcap_{P \in S} B_{P}$. This is the kernel of $\varphi$. Since $\prod_{P \in S} R / P^{n_{P}}$ is $S$-reduced, every $S$-divisible submodule of $A$ must be contained in $D$. We prove that $D$ is $S$-divisible and so it is the largest $S$-divisible submodule of $A$.

If $P \in S$, then $B_{P} / D=\prod_{Q \in S \backslash\{P\}} R / Q^{n_{Q}}$ is $P$-divisible. In other words, $B_{P}=D+P B_{P}$ and hence the localization of $P$ at $D$ is $B_{P}$, and so $P$-divisible. Hence $D$ itself is $P$-divisible. All in all, $D$ is $P$-divisible for all $P \in S$ and so $D$ is $S$-divisible.

So far we have proved (2) except that $A / D$ is $S$-pure in $\prod_{P \in S} R / P^{n_{P}}=\Pi$. Since $T$ is $S$-pure in $\Pi$ and $\Pi / T$ is $S$-divisible, this is equivalent to saying that $(A / D) / T$ is $S$-divisible. But already $A / T$ is $S$-divisible as can be easily seen: for $P \in S$, the factor $A / T$ is $P$-divisible, being a factor of the $P$-divisible $B_{P}$ by (11). 
3.2. Non-reduced $E(R)$-algebras. We prove Theorem 1.3 The methods are similar to the proof of Theorem[1.1(1).

Proof of Theorem 1.3. Let $A$ be a non-reduced $E(R)$-module. Its torsion part must be reduced by Theorem 1.1(1). It follows that the divisible part must be $Q^{(\kappa)}$ for some $\kappa \geq 1$. By Lemma 3.1, if $\kappa>1$, then $Q^{(\lambda)}$ is a direct summand of $A$ for some $\lambda>\kappa$. This is impossible, and therefore the divisible part of $A$ is $Q$. So $A$ splits as $A=Q \oplus C$ for some reduced module $C$. We have

$$
A \cong \text { Endo } A=\text { Endo } Q \oplus \operatorname{Hom}(Q, C) \oplus \operatorname{Hom}(C, Q) \oplus \text { Endo } C \text {. }
$$

The first three summands are divisible and Endo $Q \cong Q$. Since the divisible part of $A$ is only $Q$, the second and third summands must be zero. In particular, $\operatorname{Hom}(C, Q)=0$ means $C$ is torsion, so $C$ is the torsion part of $A$. Hence the torsion part $C$ of $A$ is a direct summand, so $C$ is cyclic as shown in Subsection 2.2

\section{Construction of mixed E-Rings}

The next lemma is our main tool for constructing mixed (generalized) $E(R)$ algebras out of torsion-free ones:

Lemma 4.1. Let $S$ be an infinite set of prime ideals and $B$ an algebra such that

$$
T:=\bigoplus_{P \in S} R / P^{n_{P}} \subseteq_{S} B \subseteq S \prod_{P \in S} R / P^{n_{P}}=: \Pi .
$$

Moreover, let $C$ be an $S$-divisible, torsion-free generalized $E(R)$-algebra. Fix an $R$-algebra isomorphism $\phi: C \cong$ End $C$. Let $D$ be an ideal of $C$ such that $C / D \cong$ $B / T$. Suppose that $D$ is invariant under endomorphisms and that the induced map $C \cong$ End $C \rightarrow$ End $C / D$ maps every $c \in C$ to the multiplication by the residue class of $c$. (Note that $C / D \cong B / T$ is commutative.)

Then the pull-back algebra $A$ defined by the next diagram is a generalized $E(R)$ algebra.

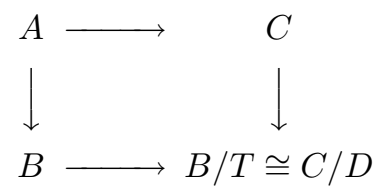

$A$ is commutative exactly if $C$ is commutative.

Proof. Note that the pull-back diagram (16) extends to a diagram of exact sequences of $R$-modules:

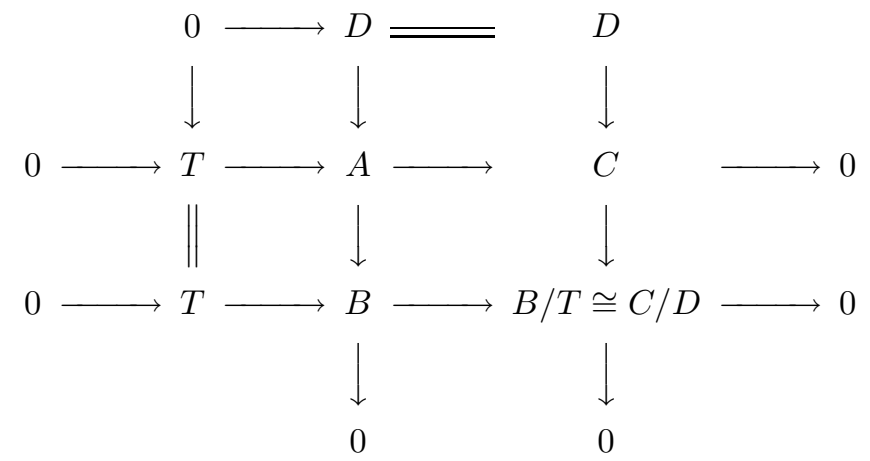


Therefore we can regard $T$ and $D$ as $R$-submodules of $A$; they are even ideals. Since $B \cong A / D$ is $S$-reduced and $D$ is $S$-divisible (as an ideal of the $S$-divisible algebra $C), D$ is the largest $S$-divisible submodule of $A$. Similarly, since $T$ is torsion and $C \cong A / T$ is torsion-free, $T$ is the torsion part of $A$.

We can look at diagram (16) as a pull-back diagram of algebras of endomorphisms: $B$ resp. $B / T$ act on themselves via multiplication and $C$ acts on itself via $\phi$. The conditions of the lemma guarantee the compatibility of these actions with the maps of (16). Hence, by pull-back, $A$ is isomorphic to the subalgebra of End $A$ consisting of those endomorphisms which induce an endomorphism on both $B$ and $C$ i.e. under which $D$ and $T$ are invariant. But this subalgebra is clearly the whole End $A$ since every endomorphism of $A$ must leave the torsion part $T$ and the largest $S$-divisible submodule $D$ invariant. Thus $A$ is a generalized $E(R)$-algebra. It is obvious from the pull-back diagram (16) that $A$ is commutative exactly if $C$ is commutative.

Proof of Theorem 1.2. We are going to apply Lemma 4.1 to obtain the required algebras.

By the localization of an $R$-module $M$ at a set $W$ of some prime ideals we mean the $R_{W}$-module $M_{W}:=M \otimes_{R} R_{W}$. Note that $M_{W}$ is an $R_{W}$-algebra if $M$ is an $R$-algebra.

Let $P$ be any prime ideal not in $S$. Choose any algebra $B_{0}$ as in (15), which is $P$-reduced and $E:=B_{0} / T$ is $P$-cotorsion-free. (Take e.g. the $B_{0}$ determined by $B_{0} / T=R_{S}$.)

Since $E$ is $P$-cotorsion-free, there is a set $X$, larger than any given cardinal, and an $E(R)$-algebra $C$ such that

$$
E[X] \subseteq C \subseteq E[X]_{P}=E_{P}[X]
$$

where $E[X]$ denotes the polynomial $R$-algebra over $E$ in the set of variables $X$. (Such a $C$ can be constructed in the following way. Let us start with a polynomial algebra $C_{0}:=E\left[X_{0}\right]$. In the $P$-adic completion of $C_{0}$, we choose an appropriate set $X_{1}$ of independent elements over $C_{0}$. Let $X:=X_{0} \cup X_{1}$ and $C$ be the purification of $C_{0}\left[X_{1}\right]=E[X]$ in the $P$-adic completion of $C_{0}$. See [1, 3, 4] for more details.)

We view the elements of $C$ as polynomials in $E_{P}[X]$. Let $D$ be the ideal of $C$ consisting of polynomials with zero constant term, i.e. the kernel of the algebra homomorphism which substitutes 0 for every variable. Thus $E \subseteq C / D \subseteq E_{P}$. Since $\Pi / T$ is a divisible torsion-free algebra, we can identify $E_{P}$, and hence $C / D$, as a subalgebra $B / T$ of $\Pi / T$ for some subalgebra $B$ of $\Pi$.

Now let us apply Lemma 4.1 for the data $B, T, C$ and $D$. The result is an $E(R)$ algebra $A$ such that $A / D \cong B$ and $A / T \cong C$. We prove that $D$ is not a direct summand of $A$. If it were a direct summand, then the projection onto $D$ would be a multiplication by some $p \in A$ with $p^{2}=p$ (since $A$ is an $E(R)$-algebra). Since the image of multiplication by $p$ is $D$, we have $p=p \cdot 1 \in D$. So, by $D \subseteq E[X]_{P}$, the element $p$ would be a non-zero idempotent polynomial with zero constant term. But no such polynomial exists.

\section{REFERENCES}

[1] Manfred Dugas, Adolf Mader, and Charles Vinsonhaler, Large E-rings exist., J. Algebra 108 (1987), 88 - 101. MR0887193 (88e:16047)

[2] Theodore G. Faticoni, Each countable reduced torsion-free commutative ring is a pure subring of an E-ring., Commun. Algebra 15 (1987), 2545 - 2564. MR0917754 (88i:20078) 
[3] Rüdiger Göbel and Jan Trlifaj, Endomorphism algebras and approximations of modules, Walter de Gruyter Verlag, Berlin, to appear, 2005.

[4] Rüdiger Göbel and Simone L. Wallutis, An algebraic version of the strong black box, Algebra and Discrete Mathematics, 1 (3) (2003) 7 - 45. MR2048638

[5] P. Schultz, The endomorphism ring of the additive group of a ring., J. Aust. Math. Soc. 15 (1973), 60 - 69. MR0338218(49:2984)

Alfréd Rényi Institute of Mathematics, Hungarian Academy of Sciences, Budapest, REÁltanoda u 13-15, 1053 Hungary

Fachbereich 6, Mathematik, Universität Duisburg-Essen, Universitätsstrasse 3, 45117, Germany 